\title{
Prediction method of favorable area of tight sandstone gas_— Taking Hechuan area as an example
}

\author{
JIANG LIN ${ }^{1}$ \\ ${ }^{1}$ Research Institute of Petroleum Exploration and \\ Development, CNPC, Beijing, 100083
}

The geological characteristics of tight sandstone gas are complex, which seriously restricts its exploration and development efficiency. It is necessary to start from the study of hydrocarbon accumulation mechanism, through the dissection of the actual regional hydrocarbon accumulation formation process, to reveal the main control factors of gas saturation of tight sandstone reservoir, and effectively guide the exploration and development of tight sandstone gas. This study analyzes the geological characteristics of tight sandstone gas accumulation in Hechuan gas field, Sichuan Basin, and reveals the main control factors of tight sandstone gas accumulation. The comprehensive analysis shows that the main controlling factors of gas saturation of tight sandstone reservoir include two aspects: one is the external factor, which is the pressure difference necessary for the formation of natural gas filling; the other is the internal factor, which is the development of fractures and coal lines in tight sandstone reservoir system, as well as the porosity, permeability and wettability of reservoir. On the basis of a large number of experimental tests and analysis, through data statistical analysis, it is found that the gas saturation is directly proportional to the filling pressure, and the gas saturation under different filling pressure is directly proportional to ratio of reservoir permeability to porosity. The results of gas testing are superimposed on the isopleth map of overpressure and the isopleth map of ratio of reservoir permeability to porosity. It is found that the area with overpressure of over $7 \mathrm{MPa}$ and the area with ratio of reservoir permeability to porosity greater than 0.02 are just the relatively high-yield areas. Hechuan area is a relatively mature area for exploration and development of tight sandstone gas. The overpressure (filling pressure) and ratio of reservoir permeability to porosity superposition area are consistent with the relatively high-yield area, so we can use these two parameters to predict the unknown area. However, because the two parameters of filling pressure and ratio of reservoir permeability to porosity must be obtained by drilling, this method needs to be determined by a certain number of exploration wells and evaluation wells in the area to gradually divide the favorable area for more efficient development. 